\title{
Performance analysis of the six-phase induction machine based on trapezoidal phase current waveform
}

\author{
Fuhui-kai ${ }^{1, a}$ \\ ${ }^{1}$ Mechanical and Electrical Engineering Institute, Xinxiang University, Xinxiang 453003, China \\ afuhuikai@163.com
}

Keywords: six-phase induction machine; Trapezoidal wave phase current; Air gap flux linkage; Electromagnetism torque;

\begin{abstract}
With the characteristic which the control of the multiphase machine is more complex compared with three-phase ac machine, a novel control method is proposed in this paper, namely six-phase motor trapezoidal wave phase current control. By using the trapezoidal phase current waveform, the stator winding is separated into the field winding and the torque winding. The function which is about the field and torque control in directed and separated mode can be realized without the complex Park transformation. The paper carries on the theoretical analysis, the computation of the air gap magnetomotive force (MMF), the electromagnetism torque and MMF decoupling parameter $\mathrm{k}$. And these results are validated by the experiment. It is shown from the theoretical analysis computation and experiment result that it is possible for the control strategy proposed in this paper and it is also of some advantages not only in the control method but also for motor control performance.
\end{abstract}

\section{Introduction}

Ac machine drives have progressed quickly, because of the inherent defects of dc machine. It has been shown that high phase order (HPO) induction machine drives possess many advantages in some special applications. These advantages include the reduction of the amplitude and increase of the frequency of the torque pulsation, the reduction of rotor harmonic currents, the reduction of the current per phase without increasing the voltage per phase and the provision of a higher reliability[1,2].

The character and development of six-phase induction machine control is introduced and summarized, and a novel control method is proposed in this paper, namely six-phase motor trapezoidal wave phase current control. By using the trapezoidal phase current waveform, the stator winding is separated into the field winding and the torque winding. The function which is about the field and torque control in directed and separated mode can be realized without the complex Park transformation[3-7].

\section{Six-phase current waveform configuration}

The stator phase current waveforms can be configured, as shown in Fig.1.The current waveforms produce a rectangular flux density in the air gap. The field and torque current components, $I_{\mathrm{F}}$ and $I_{\mathrm{T}}$ can be controlled separately like in a dc machine. The control system is simple to implement, because unlike in vector control, there are not any transformations. 


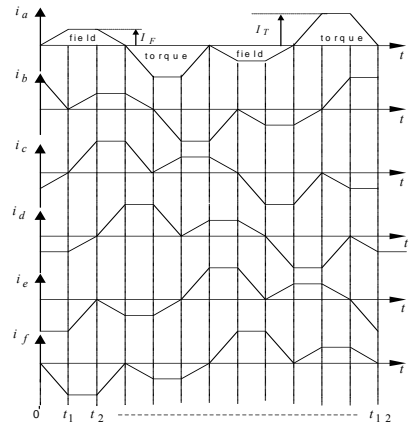

Fig.1 Six-phase current waveform configuration

The phase current waveforms are assumed to be supplied by six full-bridge converters. With these current waveforms two separate rotating stator MMFs are generated, namely a field rotating MMF and a torque rotating MMF.

$F_{f}$ is the field MMF due to the three-phase field currents, $i_{a}, i_{c}$ and $i_{d} . F_{t}$ is the torque MMF due to the three-p hase torque currents, $i_{b}, i_{e}$ and $i_{f} . F_{r}$ is the rotor MMF due to the rotor phase induced currents $i_{7}, i_{8}, \ldots, i_{13}$.

\section{The air gap field intensity calculation and analysis}

In Fig. 2 the waveforms of the three phase currents used to generate the field MMF are redrawn for time $t=0-t_{1}$.The instantaneous values of the three phase currents can be expressed as,

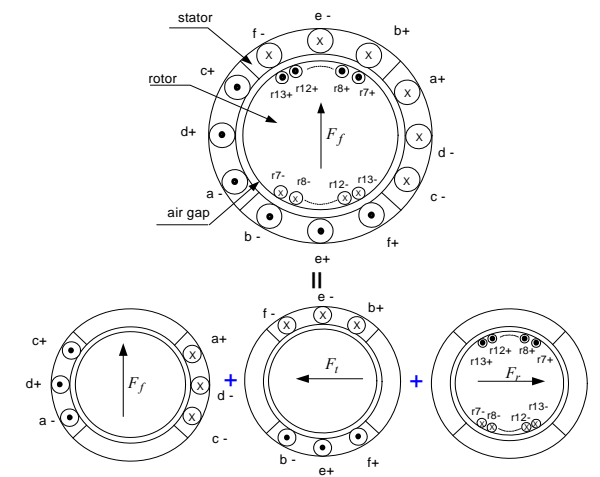

Fig.2 MMF configuration inside the machine at $t=t_{1 / 2}$

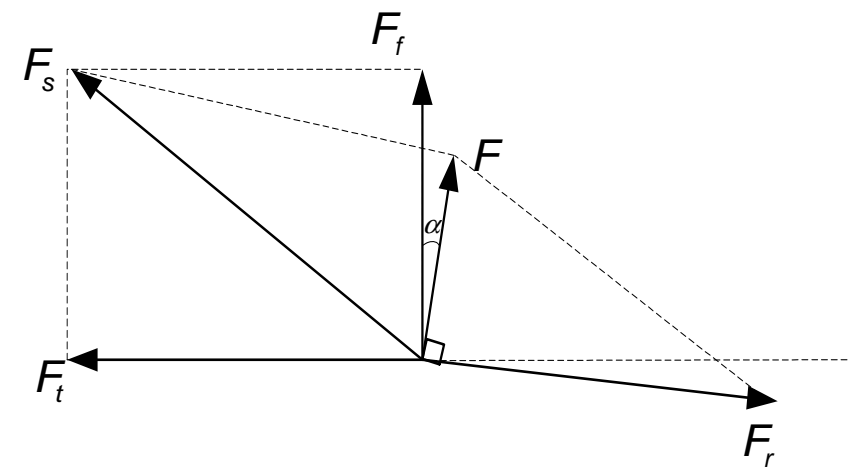

Fig.3 MMF phasor composition diagram

$$
\begin{gathered}
i_{a}=\frac{I_{F}}{t_{1}} t \\
i_{c}=\frac{I_{F}}{t_{1}} t-I_{F} \\
i_{d}=-I_{F}
\end{gathered}
$$

From this the resultant amplitude of the field MMF, $F_{f}$, for time interval $0-t_{1}$ can be calculated as,

$$
F_{f}=N_{a} i_{a}-N_{c} i_{c}-N_{d} i_{d}=2 N_{s} I_{F}
$$

For the stator torque currents during time $t=0-t_{1}$, as shown in Fig.1, the amplitude of the torque MMF, $F_{t}$ can be expressed as,

$$
F_{t}=N_{b} i_{b}-N_{e} i_{e}-N_{f} i_{f}=2 N_{s} I_{T}
$$

With seven (of the fourteen) rotor phases active for this machine, the amplitude of the rotor MMF, $F_{r}$, can be expressed as,

$$
F_{r}=\sum_{i=7}^{13} N_{r i} i_{r i}=7 N_{r} I_{r}
$$

For balanced MMF condition, $F_{t}=F_{r}$ and a $=0$ in Fig. 3 which means that

$$
2 N_{s} I_{t}=7 N_{r} I_{r}
$$


From equation (4) thus

$$
I_{t}=\frac{7 N_{r} I_{r}}{2 N_{s}}
$$

Table 1 Design data of six-phase induction machine Table

\begin{tabular}{ll}
\hline Number of phases & 6 \\
\hline Number of poles & 2 \\
Number of stator slots & 36 \\
Number of slots per pole per phase & 3 \\
Number of turns in series per stator phase & 249 \\
Stator phase resistance $(\Omega)$ & 9.6 \\
Stack outer diameter $(\mathrm{mm})$ & 165 \\
Stack length $(\mathrm{mm})$ & 128 \\
Air gap radius $(\mathrm{mm})$ & 49 \\
Number of rotor slots & 28 \\
Number of rotor phase & 14 \\
Number of turns in series per rotor phase & 28 \\
Rotor phase resistance $(\Omega)$ & 0.43 \\
\hline
\end{tabular}

The important relationship between $I_{t}$ and $\omega_{s l}$ is obtained namely,

$$
k=\frac{\omega_{s l}}{I_{t}}=\frac{I_{r}}{I_{t}} \frac{R_{r}}{2 N_{r} B L r_{g}}=\frac{N_{s} R_{r}}{7 N_{r}^{2} B L r_{g}}
$$

The design data of the machine are given in Table 1 a typical value of $\mathrm{k}=7 \mathrm{rad} /(A \cdot \mathrm{s})$ must be used in the drive control system to have decouple control.

From Fig. 3 it follows that:

$$
F^{2}=F_{s}^{2}-F_{r}^{2}=F_{f}^{2}+F_{t}^{2}-F_{r}^{2}
$$

By ignoring the stator and rotor core reluctances, the air flux density can be expressed as,

$$
\begin{aligned}
B & =\mu_{0} \frac{F}{2 l_{g}} \\
& =\mu_{0} \frac{\sqrt{\left(2 N_{s} I_{F}\right)^{2}+\left(2 N_{s} I_{T}\right)^{2}-\left(7 N_{r} I_{r}\right)^{2}}}{2 l_{g}}
\end{aligned}
$$

Table 2 Calculated result s of flux density

\begin{tabular}{lll}
\hline$I_{F}(\mathrm{~A})$ & $B(\mathrm{~T})$ & $F_{\text {air }}(\mathrm{At})$ \\
\hline 0.22 & 0.05 & 46.5 \\
0.45 & 0.1 & 93 \\
0.88 & 0.2 & 186 \\
1.32 & 0.3 & 279 \\
1.86 & 0.4 & 372 \\
4.14 & 0.5 & 465 \\
\hline
\end{tabular}

\section{Finite element calculated result}

For the FE analysis, the mesh generation is completed as shown in Fig.4. The different values of the amplitude of the flux density in the air gap can be obtained by changing $I_{F}$. The result of the air gap flux density calculation at $I_{F}=3.5 \mathrm{~A}$ is shown in Fig.5. The flux density waveform obtained from FE analysis is filtered by a low pass filter in Matlab software. 


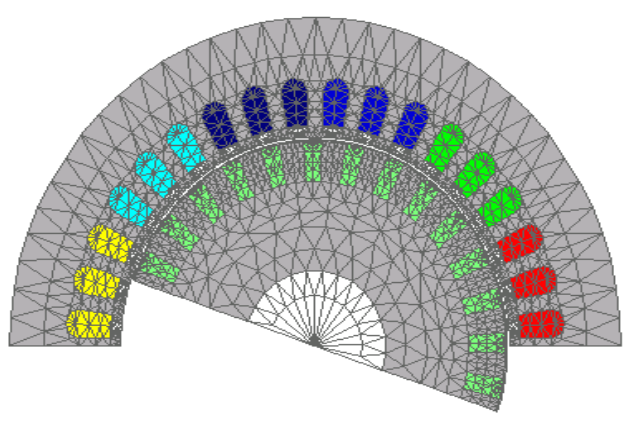

Fig.4 Mesh used in the FE analysis

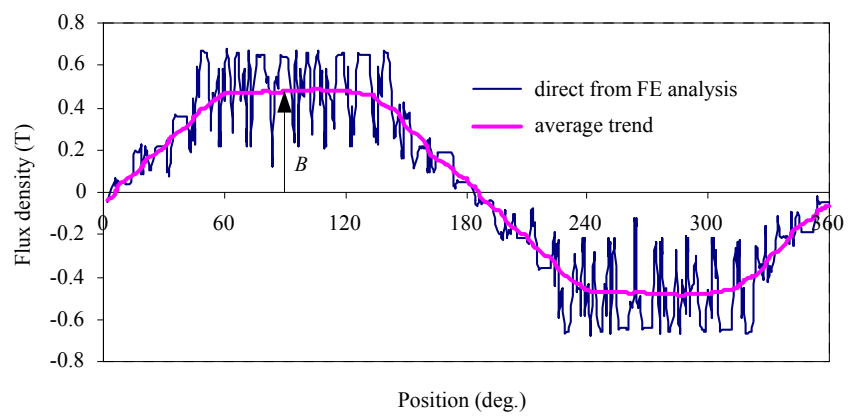

Fig.5 Flux density distribution in the air gap

\section{Experimental results}

The flux density can be estimated by modifying Eqs. (11) as

$$
B=\frac{E}{2 N_{r} l \omega_{s l} r}
$$

With $\mathrm{E}$ and $\omega_{s l}$ measured, the results of flux density versus field current are shown in Fig.6.Also shown in Fig.6 are the theoretical and FE calculated results which show fairly good agreement.

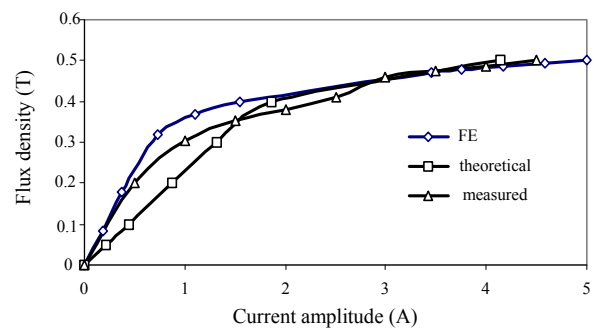

Fig.6 Comparison of results of air gap flux density versus field current IF

\section{Conclusions}

1) This novel current control strategy benefits the developed torque of the motor as well as simplifying the control algorithm by removing the complex transformations used in flux orientated control.

2) The resultant field intensity due to the field MMF in the air gap is approximately trapezoidal, which is similar to the air gap field intensity in a dc motor. The simulation of DC motor control is realized really.

3) In general good agreement is found between calculated and measured results of the proposed six-phase machine drive with its novel phase current control.

\section{References}

[1] Ai Yongle, Kamper M.J., Le Roux A. D.. Novel direct flux and direct torque control of six-phase induction machine with nearly square air-gap flux density. IEEE Trans.on Ind. Appl.Vol.43. 2007,1534-1543.

[2] Ai Yongle, Wang Yumei, Kamper M. J.. Modeling and control of six-phase induction machine under special current waveform. Iternational Journal Modelling, Identification and Control, Vol.45 No.8 (2009), pp1-8.

[3] D.W. Novotny, T.A. Lipo. Vector control and dynamics of AC drives. [J] Oxford University Press Inc.. Oxford. New York. 1997. 
[4] Yongle Ai, M.J.Kamper, Yumei Wang, Shiying Yuan. Torque Performance Investigation of Double Three-Phase Motor Using Special Current Waveform,[C] Proc. 4th conf.2004 International Electronic Motion Control ( IPEMC2004) Xi'an China, pp. 1673 1678.

[5] Ai Yongle, Wang Yumei, Kamper M. J..FPGA Application in the Six-phase Machine with Novel Control Strategy. 2008 Proceeding of Information Technology and Environmental System Sciences. Jiaozuo China.

[6] G. K. Singh, K. Nam, and S. K. Lim, "A simple indirect field oriented control scheme for multiphase induction machine", IEEE Trans. Industrial electronics, 52(4), pp. 1177-1184, (2005).

[7] Ai Yongle. Novel direct flux and direct torque control of six-phase induction machine with special current waveform. Ph.d. dissertation(2006.10). Stellenbosch University 